\title{
Infestation de Gobio gobio L. (Téléostéen Cyprinidae) par un Protozoaire énigmatique
}

\author{
Par Lucie ARVY et Guy MOREAU
}

En disséquant des Gobio gobio L. pêchés dans la Dordogne, à proximité du bourg de Siorac, nous avons remarqué dans deux cas (chez un mâle et une femelle) un aspect anormal de la rate. Chez ces deux goujons, les rates étaient légèrement hypertrophiées et, au lieu d'avoir leur teinte rouge violacée uniforme habituelle, elles étaient parsemées de macules beige rosâtre, de formes et de tailles irrégulières. Nous avons cherché à préciser les caractères de cette atteinte splénique, sur coupes histologiques, après fixation par le liquide de Bouin et inclusion à la paraffine. Nous avons pu effectuer deux colorations d'intérêt topographique (hémalun de Masson, picro-indigo carmin et trichrome en un temps), ainsi que quatre colorations d'intérêt plus précisément histochimique [bleu de toluidine, Feulgen, acide périodique Schiff (= A.P.S.) et lugol, avec épreuves de contrôle par l'amylase salivaire pour ces deux dernières].

En fait, les taches anormales, qui avaient retenu notre attention lors de l'examen macroscopique, apparaissent, à l'examen microscopique, comme formées d'amas de kystes (fig. 1 à 3), plus ou moins nombreux, disséminés irrégulièrement dans le tissu splénique, avec une prévalence marquée à la périphérie de certains vaisseaux (fig. 4). Les amas sous-capsulaires ne sont pas rares; ils soulèvent à peine la capsule et parfois dépriment la surface de la rate. Au niveau du hile splénique, un amas de tissu hépatique adjacent contient également des parasites, l'infestation était donc spléno-hépatique, l'atteinte du foie étant inapparente en raison de la teinte ocrée de cet organe chez nos Gobio.

En outre, l'infestation était certainement générale, car il n'est pas rare de trouver des sections de vaisseaux contenant des parasites parmi les érythrocytes.

Les amas parasitaires sont de tailles très variées (fig. 1) ; certains ne comprennent que quelques kystes, mais d'autres occupent une part importante du tissu splénique (fig. 1). Celui-ci ne manifeste aucune réaction particulière, il n'existe pas d'hypertrophie des splénocytes, ni de réaction conjonctive, ni d'afflux leucocytaire, ni de surcharge d'ocrocytes ; le parasite semble être bien toléré, à ceci près qu'il n'est pas rare de trouver des macrophages chargés de débris d'origine parasitaire. 
Le parasite lui-même est complexe. Il est formé par des kystes le plus souvent subsphériques $(19 \mu \times 19 \mu)$, parfois ovoïdes $(16 \mu \times 22 \mu)$, parfois anguleux par pression réciproque ; chaque kyste contient quatre formations proches de l'hémisphère, de $12 \mu$ de diamètre ; chacune d'elles est bordée par deux corps falciformes nucléés ; elles sont remplies de minuscules gemmules de $2 \mu$ de diamètre, sphériques, à noyau central.

Après coloration topographique, les gemmules apparaissent, grâce à leur minuscule noyau bien coloré par l'hémalun ou par le Feulgen et à leur cytoplasme réfringent (fig. 5 et 6). Après coloration par l'A.P.S., par le lugol ou par le bleu de toluidine, ces gemmules sont électivement mises en évidence, car, d'une part, leur cytoplasme est riche en substance basophile, électivement colorable par le bleu de toluidine et, d'autre part, il est extrêmement riche en un polysaccharide coloré en rouge brun par le lugol et en rouge vif par l'A.P.S. (fig. 7) ; ces deux dernières colorations ne pouvant plus être réalisées, quand les coupes ont été soumises à l'action de l'amylase salivaire à $37^{\circ} \mathrm{C}$ pendant une heure.

Le polysaccharide cytoplasmique des gemmules est donc du glycogène ; il est uniformément répandu dans le cytoplasme et son abondance permet d'identifier parfaitement les gemmules libres, errantes dans le tissu splénique et dans le sang, comme elle permet d'identifier, au sein des macrophages, les gemmules qu'ils ont phagocytées. L'ensemble hémisphérique avec ses gemmules rappelle l'aspect d'une demi-grenade mûre.

\section{Discussion :}

L'atteinte splénique par ce parasite énigmatique est apparemment peu fréquente ; une recherche attentive ne nous a permis de la déceler que chez deux parmi 166 goujons examinés.

Le parasitisme de la rate des Poissons n'a été que rarement observé ; seuls quelques auteurs ont, à la fin du siècle dernier, décrit quelques parasites qu'ils classaient dans le vaste groupe des Psorospermies.

Thelohan (1892) a décrit une Goussia minuta dans la rate de Tinca fluviatilis et Laveran (1897) a dénommé Coccidium metchnikovi un Sporozoaire observé dans la rate de Gobio gobio. Dans ces deux cas, les parasites étaient des Coccidies avec lesquelles le parasite que nous avons observé n'a que de lointaines analogies. Le parasite splénique vu par Laveran, chez Gobio, était particulièrement chromophobe, puisqu'il ne se colorait ni par l'éosine, ni par le bleu de méthylène, ni par la thionine phéniquée, ni par le violet de gentiane, ni par l'hématéine, et que l'eau iodée le colorait en jaune

FIG. 1 et 2. - Vues d'ensemble des rates parasitées: 1) après coloration par l'hémalun de Masson picro-indigo-carmin ; 2) après coloration trichrome en un temps. FIG. 3. - Les kystes foisonnent en bordure de la lumière d'un vaisseau. FIG. 4. - Aspect des kystes; le tissu splénique ne manifeste aucune réaction particulière au voisinage du parasite. FIG. 5 et 6 . - Après coloration trichrome en un temps, les formations camérulaires hémisphériques, riches en gemmules sont bien mises en évidence. FIG. 7. - Après coloration par l'acide periodique-Schiff, les gemmules sont électivement mises en évidence grâce à leur teneur en un polysaccharide ne résistant pas à l'amylase. 


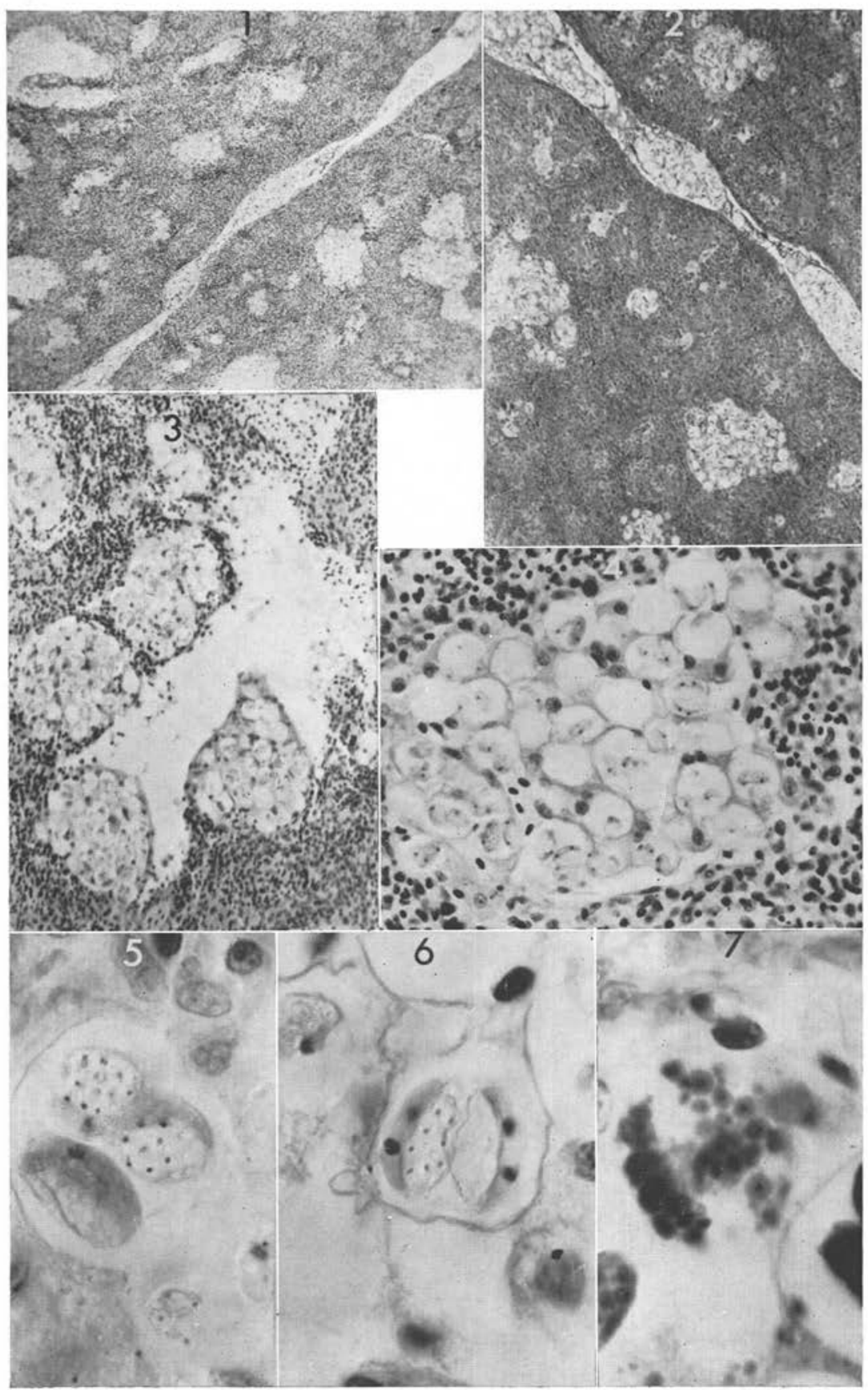


pâle. Notre parasite est au contraire très chromophile. La description de Laveran ne s'accompagne d'aucun dessin et reste d'autant plus imprécise qu'il s'agissait d'une parasitose mixte avec des Myxosporidies; néanmoins, il s'agissait indubitablement d'une Coccidie, puisque le parasite était constitué par des oocystes (de 20 à $25 \mu$ ) se divisant longitudinalement pour donner deux sporozoïtes.

Les kystes de notre parasite contiennent quatre formations camérulaires bordées par deux corps falciformes associés ; chaque chambre contient de nombreuses gemmules et les coupes favorablement orientées nous ont permis d'en compter jusqu'à 27 ; le noyau bien colorable par l'hémalun et par le Feulgen est central et mesure approximativement $0,5 \mu$; ces gemmules sont infestantes: on les retrouve dans le tissu splénique et dans le sang.

Il n'est pas possible, avec les données que nous possédons actuellement, d'attribuer une position systématique précise à ce parasite.

(Laboratoire du C.N.R.Z., à Jouy-en-Josas

et Station biologique des Eyzies) 